\title{
Adaptive stepsize implementations of implicit stochastic Runge Kutta methods with modified Wiener increment
}

\author{
R. Herdiana* K. Burrage ${ }^{\dagger}$
}

(Received 8 August 2003, revised 9 January 2004)

\begin{abstract}
An adaptive stepsize algorithm is implemented on a stochastic implicit strong order 1 method, namely a stiffly accurate diagonal implicit stochastic Runge-Kutta method where a modified Wiener increment $\Delta \bar{W}_{n}$ is involved instead of a regular $\Delta W_{n}=\beta \sqrt{h}, \beta \sim N(0,1)$ to avoid unboundedness. The modified Wiener increment is equal to the regular one only if $|\beta| \leq A_{h}$, otherwise $\Delta \bar{W}=-A_{h} \sqrt{h}$ or $A_{h} \sqrt{h}$ if $\beta<-A_{h}$ or $\beta>A_{h}$, respectively. The parameter $A_{h}$ is determined based on a strong order inequality requirement (Milstein et al., 2002)

$$
E(\bar{\beta}-\beta)^{2} \leq h^{k}, \quad k \geq 1 .
$$
\end{abstract}

${ }^{*}$ Faculty of Computer Science, University of Indonesia, Depok 16424. mailto:ratna@cs.ui.ac.id

${ }^{\dagger}$ Department of Mathematics, University of Queensland, Australia 4072. mailto:kb@maths.uq.edu.au

See http://anziamj.austms.org.au/V45/CTAC2003/Herd for this article, (C) Austral. Mathematical Soc. 2004. Published July 1, 2004. ISSN 1446-8735 
The variable stepsize algorithm is based on Richardson's extrapolation. For every step change additional work is required to compute $\Delta \bar{W}_{n}$ so that the correct Brownian path is maintained from simulation to simulation. Numerical experiments in solving nonlinear and linear stochastic differential equation problems demonstrate that better approximations are obtained with the modified Wiener increment compared to using the regular Wiener increment.

\section{Contents}

1 Introduction

2 Variable stepsize algorithm

C581

3 Modified Wiener increment

C582

4 SADISRK2 method

C585

5 Numerical experiments

C586

6 Conclusions

C590

References

C590

\section{Introduction}

A variable stepsize implementation is introduced for the strong solution of stiff stochastic differential equations (SDEs) in the Stratonovich form governed by a single Wiener process

$$
d y(t)=f(y(t)) d t+g(y(t)) \circ d W(t), \quad y \in \mathbb{R}^{m}, \quad y(0)=y_{0},
$$


where $f$ and $g$ are real valued functions called the drift and diffusion coefficients, respectively; and $W(t)$ is a scalar Wiener process. Stiff sDEs are solved using stochastic implicit methods. A major problem in constructing stochastic implicit methods is the possibility of obtaining unrealized (unbounded) solutions due to the nature of the Wiener process. To avoid unboundedness Milstein et al. [6] introduced a modified Wiener increment $\Delta \bar{W}=\bar{\beta} \sqrt{h}$ to approximate $W(t)$, where

$$
\bar{\beta}=\left\{\begin{array}{cl}
\beta, & \text { if }|\beta| \leq A_{h}, \\
-A_{h}, & \text { if } \beta<-A_{h}, \\
A_{h}, & \text { if } \beta>A_{h} .
\end{array}\right.
$$

For $A_{h}=\sqrt{2 k|\ln h|}(k \geq 1)$, the strong inequality

$$
E(\bar{\beta}-\beta)^{2} \leq h^{k}, \quad k \geq 1,
$$

is a sufficient condition to preserve the order of the method.

A stiffly accurate diagonally implicit stochastic Runge-Kutta (SADISRK2) method of strong order 1 with a modified Wiener increment was constructed by Burrage and Tian in [5] and stability analysis based on fixed stepsize implementation shows that SADISRK2 method is suitable for solving stiff SDEs.

In this paper we implement a variable stepsize mode of the SADISRK2 method. The major implementation issue is how to handle the modified Wiener increment whenever there is a stepsize change such that integration is guaranteed to remain in the correct Wiener path from simulation to simulation. The approach is based on the implementation used for regular Wiener increment.

Numerical experiments show that significant improvement in accuracy is gained when the modified Wiener is used compared to the regular Wiener. 


\section{Variable stepsize algorithm}

Richardson's extrapolation in [2] for stepsize selection is described as follows: at each integration time $t_{n}$, a starting stepsize $h_{n}$ is chosen, the program computes two numerical solutions at $t_{n}+h_{n}$. Let $y$ be the numerical result of one stepsize $h_{n}$, then this time step is repeated in two steps of size $h_{n} / 2$, denote by $\tilde{y}$. The difference $e=\tilde{y}-y$, estimates the local error $r_{n}$ and computes the next stepsize. Since in stochastic setting that the error increases by order $1 / 2$, we have

$$
r_{n}=\frac{\|e\|}{2^{p+1 / 2}-1}=\frac{1}{2^{p+1 / 2}-1} \sqrt{\frac{1}{N} \sum_{i=1}^{N}\left(\frac{e_{i}}{\mathrm{tol}^{2}}\right)^{2}},
$$

where $p$ is the order of the method, in this case $p=1$. If $r_{n} \leq 1$ the step is accepted, otherwise it is rejected and a new stepsize $h_{n+1}$ such that $e \approx$ tol which leads to

$$
h_{n+1}=\left(\frac{1}{r_{n}}\right)^{1 / p+1 / 2} h_{n} .
$$

An alternative stepsize control strategy is the predictive-PID (Proportional Integral Derivative) [7]:

$$
h_{n+1}=\left(\frac{0.8 \cdot \mathrm{tol}}{r_{n}}\right)^{k_{I}}\left(\frac{r_{n-1}}{r_{n}}\right)^{k_{P}}\left(\frac{r_{n-1}^{2}}{r_{n} r_{n-1}}\right)^{k_{D}} h_{n},
$$

where $k=p, k k_{I} \in[0.1,0.25], k k_{P}=0.45$ and $k_{D}=k_{I} / 4=-k_{P} / 4$.

In the stochastic setting, an important factor in implementing variable stepsize is how to maintain in the correct Brownian path from simulation to simulation [1, 3, 4]. Here we use an efficient approach introduced in [4] and outline the algorithm below. First, generate a fixed Brownian path with fixed stepsize $h$ and denote $j_{1}=\int_{t_{0}}^{t_{0}+h} d W(s)$. Suppose step $h_{1}<h$ is taken, then given $j_{1}$ we need to simulate $J_{1}$ on two sub-intervals $\left[t_{0}, t_{0}+h_{1}\right]$ and 
$\left[t_{0}+h_{1}, t_{0}+h\right]$. This involves using a new random variable $Z$ :

$$
J_{1}\left(t_{0}, t_{0}+h_{1}\right)=\frac{h_{1}}{h} j_{1}+Z, \quad J_{1}\left(t_{0}+h_{1}, t_{0}+h\right)=\frac{h_{2}}{h} j_{1}-Z,
$$

(where $\left.h_{2}=h-h_{1}\right)$, then it is clear that $J_{1}\left(t_{0}, t_{0}+h_{1}\right)+J_{1}\left(t_{0}+h_{1}, t_{0}+h\right)=j_{1}$ thus the sum is equal to the Wiener increment of the fixed path (that is, the same trajectory is maintained). Also the following are satisfied

$$
\begin{aligned}
& h_{1}:=E\left(\left(J_{1}\left(t_{0}, t_{0}+h_{1}\right)\right)^{2}\right)=\frac{h_{1}^{2}}{h^{2}} E\left(j_{1}^{2}\right)+E\left(Z^{2}\right), \\
& h_{2}:=E\left(\left(J_{1}\left(t_{0}+h_{1}, t_{0}+h\right)\right)^{2}\right)=\frac{h_{2}^{2}}{h^{2}} E\left(j_{1}^{2}\right)+E\left(Z^{2}\right),
\end{aligned}
$$

which leads to

$$
E\left(Z^{2}\right)=\frac{h^{2}-h_{1}^{2}-h_{2}^{2}}{2 h}=\frac{h_{1} h_{2}}{h} .
$$

Similarly the requirement that $E\left(J_{1}\left(t_{0}, t_{0}+h_{1}\right) J_{1}\left(t_{0}+h_{1}, t_{0}+h\right)\right)=0$ leads to $E\left(Z^{2}\right)=h_{1} h_{2} / h$. Therefore $Z$ is determined by sampling from $N \sim\left(0, h_{1} h_{2} / h\right)$, and this ensures that the $J_{1}$ samples on the subintervals have the correct distribution and are on the correct path when moving from simulation to simulation.

\section{Modified Wiener increment}

The implementation of a variable stepsize involving a modified Wiener process requires determining the boundary value $A_{h}$ whenever a step is changed. Let $\Delta W_{i}, i=1, \ldots, N$ be a fixed stepsize Wiener path generated initially along equidistant intervals $t_{0}<t_{1}<\ldots<t_{N}$ with stepsize $h$ where $\Delta W_{i}=$ $\xi_{i} \sqrt{h}, \xi_{i} \sim N(0,1), i=1, \ldots, N$.

There are two modifications required for every step change. First, the Wiener increment at the lower level (that is, of the fixed path); and second is 
to the actual Wiener increment for the current stepsize. Suppose at point $t_{n}$, $\Delta W_{n}=\xi_{n} \sqrt{h}$ is the regular fixed Wiener increment in $\left[t_{n}, t_{n+1}\right], t_{n+1}=$ $t_{n}+h$. The modified Wiener increment is

$$
\Delta \bar{W}_{n}=\sqrt{h} \bar{\xi}_{n}=\sqrt{h}\left\{\begin{array}{cl}
\xi_{n}, & \text { if }\left|\xi_{n}\right| \leq A_{h}, \\
-A_{h}, & \text { if } \xi_{n}<-A_{h}, \\
A_{h}, & \text { if } \xi_{n}>A_{h},
\end{array}\right.
$$

where $A_{h}=\sqrt{2 k|\ln h|}(k \geq 1)$, and from (3) the mean-square difference is

$$
\begin{aligned}
E\left(\Delta W_{n}-\Delta \bar{W}_{n}\right)^{2} & =E\left(\sqrt{h}\left(\xi_{n}-\bar{\xi}_{n}\right)\right)^{2} \\
& =h E\left(\xi_{n}-\bar{\xi}_{n}\right)^{2} \leq h^{k+1}
\end{aligned}
$$

Suppose a new stepsize $h_{1}<h$ is desired: let $h_{2}=h-h_{1}$, and denote $h^{*}$ as the sum of $h_{1}$ and $h_{2}$ (at this point $h^{*}=h$ ). The Wiener increments for the new subintervals $\left[t_{n}, t_{n}+h_{1}\right]$ and $\left[t_{n}+h_{1}, t_{n+1}\right]$ are, respectively,

$$
\Delta W_{n 1}=\frac{h_{1}}{h^{*}} \Delta W_{n}+Z, \quad \Delta W_{n 2}=\frac{h_{2}}{h^{*}} \Delta W_{n}-Z ;
$$

where $Z=\rho \sqrt{\frac{h_{1} h_{2}}{h^{*}}}, \rho \sim N(0,1)$ thus $Z \sim N\left(0, \frac{h_{1} h_{2}}{h^{*}}\right)$. The modifications of the above increments are, respectively,

$$
\Delta \bar{W}_{n 1}=\frac{h_{1}}{h^{*}} \Delta \bar{W}_{n}+\bar{Z}, \quad \Delta \bar{W}_{n 2}=\frac{h_{2}}{h^{*}} \Delta \bar{W}_{n}-\bar{Z} .
$$

The new random variable $\bar{Z}=\bar{\rho} \sqrt{h_{1} h_{2} / h^{*}}$, where

$$
\bar{\rho}=\left\{\begin{array}{cl}
\rho, & \text { if }|\rho| \leq A_{H}, \\
-A_{H}, & \text { if } \rho<-A_{H}, \\
A_{H}, & \text { if } \rho>A_{H},
\end{array}\right.
$$

and $A_{H}$ is determined from the mean-square difference condition

$$
E(Z-\bar{Z})^{2} \leq\left(\frac{h_{1} h_{2}}{h^{*}}\right)^{k+1}
$$


This is equivalent to

$$
\begin{aligned}
\frac{h_{1} h_{2}}{h^{*}} E(\rho-\bar{\rho})^{2} & \leq\left(\frac{h_{1} h_{2}}{h^{*}}\right)^{k+1}, \\
E(\rho-\bar{\rho})^{2} & \leq\left(\frac{h_{1} h_{2}}{h^{*}}\right)^{k} .
\end{aligned}
$$

Since $E(\rho-\bar{\rho})^{2}<\exp \left(-A_{H}^{2} / 2\right)$, the inequality (16) is fulfilled when

$$
A_{H} \geq \sqrt{2 k\left|\ln h_{1} h_{2} / h^{*}\right|} .
$$

In order to remain in the same Brownian path, the mean and mean-square sums must satisfy

$$
\begin{aligned}
E\left[\Delta W_{n 1}-\Delta \bar{W}_{n 1}\right]+E\left[\Delta W_{n 2}-\Delta \bar{W}_{n 2}\right] & =E\left[\Delta W_{n}-\Delta \bar{W}_{n}\right] \equiv 0 ;(17) \\
E\left(\Delta W_{n 1}-\Delta \bar{W}_{n 1}\right)^{2}+E\left(\Delta W_{n 2}-\Delta \bar{W}_{n 2}\right)^{2} & \leq\left(h^{*}\right)^{k+1} .
\end{aligned}
$$

Clearly (17) is true as $E(Z-\bar{Z})=0$. It remains to show (18). By substituting $\Delta W_{n 1}$ and $\Delta W_{n 2}$ from (12) and their corresponding modifications (13) into the left-hand side of (18), then rearranging and following (15) and (11) we obtain

$$
\begin{aligned}
& \frac{h_{1}^{2}+h_{2}^{2}}{\left(h^{*}\right)^{2}} E\left(\Delta W_{n}-\Delta \bar{W}_{n}\right)^{2}+2 E(Z-\bar{Z})^{2} \\
\leq & \frac{h_{1}^{2}+h_{2}^{2}}{\left(h^{*}\right)^{2}}\left(h^{*}\right)^{k+1}+2\left(\frac{h_{1} h_{2}}{h^{*}}\right)^{k+1} \\
\leq & {\left[\left(\frac{h_{1}}{h^{*}}\right)^{2}+\left(\frac{h_{2}}{h^{*}}\right)^{2}\right]\left(h^{*}\right)^{k+1}+2\left(\frac{h_{1}}{h^{*}}\right)^{k+1}\left(\frac{h_{2}}{h^{*}}\right)^{k+1}\left(h^{*}\right)^{k+1} } \\
\leq & \left(\frac{h_{1}}{h^{*}}+\frac{h_{2}}{h^{*}}\right)^{2}\left(h^{*}\right)^{k+1} \leq\left(h^{*}\right)^{k+1} .
\end{aligned}
$$


$A_{h}$ also need adjustment in the case when the succeeding stepsize (after $h_{1}$ is successful) exceeds $h_{2}$. If the next step $h_{1}$ (new) satisfies $h_{2}<h_{1}$ (new) $<$ $2 h$, then two Wiener increments from the lower level are used that is, in the interval $\left[t_{n}+h_{1}, t_{n}+h\right] \cup\left[t_{n}+h, t_{n}+2 h\right]$. The corresponding Wiener increment for the combined intervals is the sum $\Delta W_{n 2}+\Delta W_{n+1}=\Delta W_{n+1}^{*}$. The modified version is $\Delta \bar{W}_{n+1}^{*}=\Delta \bar{W}_{n 2}+\Delta \bar{W}_{n+1}$ or

$$
\Delta \bar{W}_{n+1}^{*}= \begin{cases}\Delta W_{n+1}^{*}, & \text { if }\left|\Delta W_{n+1}^{*}\right| \leq \sqrt{h_{\text {new }}^{*}} A_{h_{\text {new }}^{*}} \\ -\sqrt{h_{\text {new }}^{*}} A_{h_{\text {new }}^{*}}, & \text { if } \Delta W_{n+1}^{*},<-\sqrt{h_{\text {new }}^{*}} A_{h_{\text {new }}^{*}} \\ \sqrt{h_{\text {new }}^{*}} A_{h_{\text {new }}^{*}}, & \text { if } \Delta W_{n+1}^{*}>\sqrt{h_{\text {new }}^{*}} A_{h_{\text {new }}^{*}} .\end{cases}
$$

Here $h_{\text {new }}^{*}=h_{2}+h(h$ is the fixed stepsize $)$ and $A_{h_{\text {new }}^{*}}=\sqrt{2 k\left|\ln h_{\mathrm{new}}^{*}\right|}$.

The same trajectory is maintained since the inequality condition is fulfilled:

$$
\begin{aligned}
E\left[\Delta W_{n+1}^{*}-\Delta \bar{W}_{n+1}^{*}\right]^{2} & =E\left[\Delta W_{n 2}-\Delta \bar{W}_{n 2}\right]^{2}+E\left[\Delta W_{n+1}-\Delta \bar{W}_{n+1}\right]^{2} \\
& \leq h_{2}^{k+1}+h^{k+1} \\
& \leq\left(h_{2}+h\right)^{k+1} .
\end{aligned}
$$

The interval $\left[t_{n}+h_{1}, t_{n}+2 h\right]$ is then subdivided into two subintervals with length $h_{1}$ (new) and $h_{\text {new }}^{*}-h_{1}$ (new), respectively, that is, $\left[t_{n}+h_{1}, t_{n}+h_{1}+\right.$ $h_{1}$ (new) $]$ and $\left[t_{n}+h_{1}+h_{1}\right.$ (new), $\left.t_{n}+2 h\right]$. Evaluations of the Wiener increments and their modifications - for the two subintervals - proceed as in (13).

\section{SADISRK2 method}

Consider an $s$-stage stochastic RK method with truncated Wiener process [5]

$$
\begin{aligned}
Y & =(e \otimes I) y_{n}+h(A \otimes I) g_{0}(Y)+\bar{\xi} \sqrt{h}(B \otimes I) g_{1}(Y), \\
y_{n+1} & =y_{n}+h\left(\alpha^{T} \otimes I\right) g_{0}(Y)+\bar{\xi} \sqrt{h}\left(\beta^{T} \otimes I\right) g_{1}(Y),
\end{aligned}
$$


or represented in tabular form

$$
\mid \begin{array}{ll}
h A & \bar{\xi} \sqrt{h} B \\
\hline h \alpha & \beta \bar{\xi} \sqrt{h}
\end{array}
$$

where $A$ and $B$ are $s \times s$ matrices, $Y=\left(Y_{1}, \ldots, Y_{s}\right)^{T}, \alpha^{T}$ and $\beta^{T}$ are $s$ dimensional row vectors, $e=(1, \ldots, 1)^{T}$ is a unit vector and $\bar{\xi}$ is the modified random variable. A 2-stage stiffly accurate diagonal method of strong order 1 referred to as the SADISRK2 method was constructed in [5] where the diagonal components are made equal to enhance computational efficiency. The method is symbolized by the tableau

$$
\mid \begin{array}{ll|ll}
\left(1-\frac{\sqrt{2}}{2}\right) h & 0 & \left(1-\frac{\sqrt{2}}{2}\right) \bar{\xi} \sqrt{h} & 0 \\
\frac{\sqrt{2}}{2} h & \left(1-\frac{\sqrt{2}}{2}\right) h & \frac{\sqrt{2}}{2} \bar{\xi} \sqrt{h} & \left(1-\frac{\sqrt{2}}{2}\right) \bar{\xi} \sqrt{h} \\
\hline \frac{\sqrt{2}}{2} h & \left(1-\frac{\sqrt{2}}{2}\right) h & \frac{\sqrt{2}}{2} \bar{\xi} \sqrt{h} & \left(1-\frac{\sqrt{2}}{2}\right) \bar{\xi} \sqrt{h}
\end{array}
$$

\section{$5 \quad$ Numerical experiments}

Example 1 Consider a 2-dimensional linear SDEs system given in Stratonovich form

$$
\begin{aligned}
d U(t) & =\left(A-\frac{1}{2} B^{2}\right) U(t) d t+B U(t) \circ d W, \quad t \in[0,1] \\
U(0) & =[-5,1]^{T}
\end{aligned}
$$

where

$$
A=\left[\begin{array}{cc}
-a & a \\
a & -a
\end{array}\right] \text { and } B=\left[\begin{array}{cc}
b & 0 \\
0 & b
\end{array}\right]
$$


TABle 1: Problem 1: $a=2, b=0.5$. Numerical performance of SADISRK2 method averaged over 500 trajectories. Regular Wiener vs modified Wiener.

\begin{tabular}{|c|c|c|c|c|c|c|c|c|}
\hline \multirow[b]{2}{*}{ tol } & \multicolumn{8}{|c|}{ with regular Wiener } \\
\hline & \multicolumn{2}{|c|}{$\epsilon$} & tried & fail & avg. $h$ & iter & fevals & $\mathrm{LU}$ \\
\hline $2^{-6}$ & $4.19(-2)$ & $4.67(-2)$ & 9 & 0 & 0.1111 & 54 & 324 & 27 \\
\hline $2^{-10}$ & $2.33(-2)$ & $2.59(-2)$ & 16 & 3 & 0.0776 & 95 & 555 & 48 \\
\hline $2^{-12}$ & $7.27(-3)$ & $8.05(-3)$ & 29 & 7 & 0.0461 & 175 & 1007 & 88 \\
\hline & \multicolumn{8}{|c|}{ with modified Wiener } \\
\hline tol & \multicolumn{2}{|c|}{$\epsilon$} & tried & fail & avg. $h$ & iter & fevals & $\mathrm{LU}$ \\
\hline $2^{-6}$ & $2.92(-3)$ & $3.02(-3)$ & 9 & 0 & 0.1111 & 54 & 324 & 27 \\
\hline $2^{-10}$ & $2.51(-3)$ & $2.60(-3)$ & 16 & 3 & 0.0786 & 94 & 546 & 47 \\
\hline $2^{-12}$ & $1.55(-3)$ & $1.65(-3)$ & 29 & 7 & 0.0465 & 173 & 998 & 87 \\
\hline
\end{tabular}

The system has an exact solution

$$
U(t)=P\left[\begin{array}{cc}
\exp \rho^{+}(t) & 0 \\
0 & \exp \rho^{-}(t)
\end{array}\right] P^{-1} U_{0}
$$

where $\rho^{ \pm}(t)=\left(-a-\frac{1}{2} b^{2} \pm a\right) t+b W(t)$,

$$
P=\frac{1}{\sqrt{2}}\left[\begin{array}{cc}
1 & 1 \\
1 & -1
\end{array}\right] \text { with } P^{-1}=P,
$$

and components of the coefficient matrices are set $a=2, b=0.5$. Fixed stepsize $h=0.1$ is used to generate the fixed Wiener increments; the initial stepsize is $h_{0}=0.05$. We compare numerical performances between using regular Wiener increment and the modified Wiener, the results are averaged over 500 simulations and presented in Table 1.

The numerical comparisons show that the SADISRK2 with modified Wiener increment provides better approximation for relatively the same amount of effort (work). 
TABLE 2: Example 2, $\alpha=1$ : SADISRK2 method.

\begin{tabular}{|l|lrrrr|}
\hline \multicolumn{5}{|c|}{$\sigma=0.2$, with predictive-PID control } \\
& tol & tried & fail & avg.h & iter \\
\hline Regular W & $2^{-8}$ & 152 & 36 & 0.0690 & 912 \\
Modified W & $2^{-7}$ & 139 & 12 & 0.0630 & 834 \\
\hline$\sigma=0.5$, with modified & Wiener process \\
& tol & tried & fail & avg.h & iter \\
\hline Standard & $2^{-11}$ & 1318 & 279 & 0.0077 & 7908 \\
pred.-PID & $2^{-10}$ & 1005 & 98 & 0.0088 & 6030 \\
\hline
\end{tabular}

Example 2 Consider a simplified version of a Duffing-Van der Pol oscillator written as a 2-dimensional sDE with $X_{1}$ and $X_{2}$ representing the displacement and speed, respectively,

$$
\begin{aligned}
& d X_{1}=X_{2} d t \\
& d X_{2}=\left\{X_{1}\left(\alpha-X_{1}^{2}\right)-X_{2}\right\} d t+\sigma X_{1} d W
\end{aligned}
$$

where $\sigma \geq 0$ governs the intensity of the multiplicative noise. To ensure the simulation is on the correct path, a fixed Wiener path with stepsize length 0.1 is generated initially.

Comparison tests between regular and the modified Wiener process and also between using standard error and predictive-PID controllers $\left(k k_{I}=0.1\right.$, $\left.k k_{P}=0.45\right)$ based on the same fixed Wiener path are presented below. We set the initial values $X_{1}(0)=-4 ; X_{2}(0)=0$ and parameters $\alpha=1 ; \sigma=0.2$ and 0.5 . The results displayed in Figure 1 and Table 2, are from the same fixed Wiener path.

The results from this example confirm the effectiveness of adopting the predictive-PID control approach in implementing adaptive time-stepping based on the SADISRK2 scheme with a modified Wiener increment. In the case of $\sigma=0.2$, for similar average stepsize, the modified Wiener increment needs 

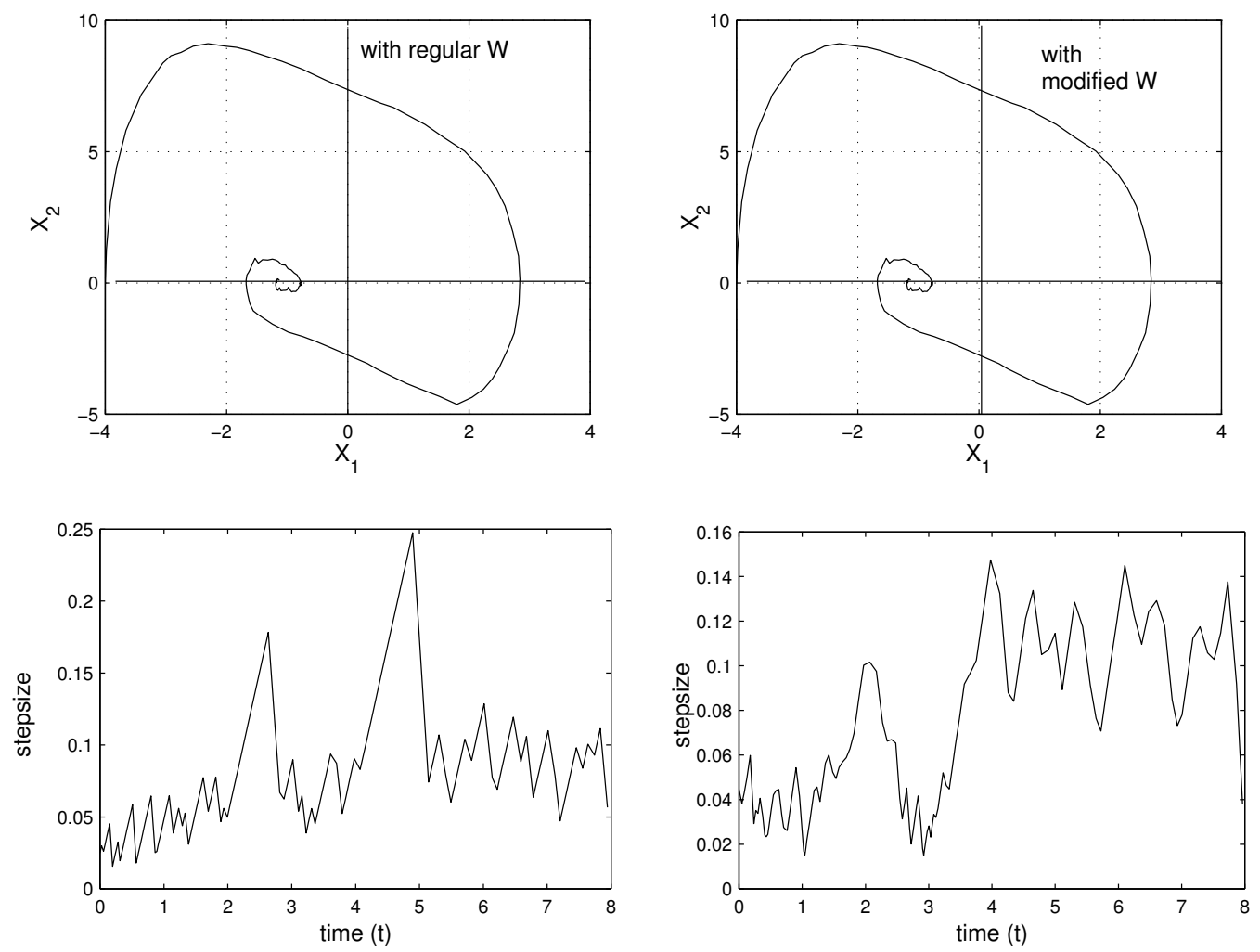

Figure 1: Example $2 \alpha=1, \sigma=0.2$. Comparison between using SADISRK2 method with regular Wiener vs modified Wiener, with predictive-PID stepsize control. Top: phase planes are almost identical. Bottom: stepsize sequence, the left is more dense. 
about $9 \%$ less steps and iterations, and $1 / 3$ of the rejected steps than using the regular Wiener.

\section{Conclusions}

We have introduced variable stepsize implementation involving a modified Wiener increment based on a 2-stage stiffly accurate stochastic RK method.

The implementation requires extra effort in determining the restriction for the modified random variables whenever there is a stepsize change, also in order for the simulations to remain in the correct Wiener path.

Numerical comparisons showed that with the modified Wiener increment we can obtain more accurate numerical solutions as opposed to the regular Wiener. Also the predictive-PID stepsize control is an alternative approach to increase efficiency.

\section{References}

[1] J. G. Gaines and T. J. Lyons. Variable stepsize control in the numerical solution of stochastic differential equations. SIAM J. Appl. Math., 5:1455-1484, 1997. C581

[2] E. Hairer, S. P. Nørsett and G. Wanner. Solving Ordinary Differential Equations I, Nonstiff Problems. Springer Verlag, Berlin, 1993. C581

[3] S. Mauthner. Stepsize control in the numerical solution of stochastic differential equations. J. of Computational and Applied Mathematics, 100:93-109, 1998. C581 
[4] P. M. Burrage and K. Burrage. A variable stepsize implementation for stochastic differential equations. SIAM J. Sci. Comput., 24:848-864, 2002. C581

[5] K. Burrage and T. H. Tian. Implicit stochastic Runge-Kutta methods for stochastic differential equations. Submitted, 2002. C580, C585, C586

[6] G. N. Milstein, YU. M. Repin and M. V. Tretykov. Numerical methods for stochastic systems preserving symplectic structure. SIAM J. Numer. Anal., 40:1583-1604, 2002. C580

[7] G. Söderlind. Digital filters in adaptive time-stepping. $A C M$ Transactions on Mathematical Software, 29(1):1-26, 2003. C581 